\title{
SEROPOSITIVITY OF IgM DENGUE (ELISA) AMONG SUSPECTED CASES OF DENGUE- A STUDY FROM TERTIARY CARE CENTRE IN PUNJAB
}

\author{
Shavi Nagpal ${ }^{1}$, Aroma Oberoi ${ }^{2}$
}

1 Postgraduate Student, Department of Microbiology, Christian Medical College and Hospital, Ludhiana, Punjab.

2Professor and HOD, Department of Microbiology, Christian Medical College and Hospital, Ludhiana, Punjab.

\section{BACKGROUND}

\section{ABSTRACT}

Dengue fever is the most common cause of arboviral disease worldwide. In India and other tropical countries, it remains an important differential diagnosis for a febrile illness. The disease burden is growing very fast in various countries including India. Aims and Objectives- This study was done to find the seropositivity of Dengue (IgM Dengue by ELISA) in blood samples taken from suspected cases of dengue fever.

\section{MATERIALS AND METHODS}

This is a hospital-based descriptive study, conducted at the serology laboratory in the Department of Microbiology at a tertiary care centre from January 2017 to December 2017. Blood samples were received in the department from indoor and outpatients who were suspected to have dengue fever. A total of 1347 samples were collected in 12 months and these samples were processed for IgM Anti-Dengue antibodies (ELISA).

\section{RESULTS}

The male-to-female ratio of suspected cases was 1.23:1. Adults constituted 855 samples, while the remaining 492 were of paediatric population. IgM Dengue ELISA was found positive in 457 samples (Positivity rate 33.92\%). Dengue positive samples were constituted by 316 adults and 141 paediatric patients giving a positivity rate of $36.95 \%$ and $28.65 \%$ respectively. The seropositivity among male and female was $35.12 \%$ and $32.45 \%$ respectively. Maximum number of dengue positive samples were detected during the month of October.

\section{CONCLUSION}

Prompt diagnosis is the key to better management and decreased morbidity/ mortality. In our study, a commercial kit (Panbio IgM Dengue ELISA) was used for diagnosis of dengue fever, which showed a seropositivity of $33.92 \%$. Various steps at multiple levels (Curb mosquito breeding, early diagnosis and treatment) are needed to stop the growing number.

\section{KEY WORDS}

Dengue, Arboviral, Febrile, ELISA.

HOW TO CITE THIS ARTICLE: Nagpal S, Oberoi A. Seropositivity of IgM dengue (ELISA) among suspected cases of dengue- a study from tertiary care centre in Punjab. J. Evolution Med. Dent. Sci. 2018;7(21):2555-2557, DOI: 10.14260/jemds/2018/575

\section{BACKGROUND}

Dengue fever is the most common cause of arboviral disease worldwide. In India and other tropical countries, it remains an important differential diagnosis for a febrile illness. The disease burden is growing very fast in tropics and various factors have been implicated for increasing disease burden. These factors include poor hygiene, broken health system and increasing international travels.[1,2]

Dengue virus (DENV) consists of four serotypes (DENV 1 to 4), is the member of flaviviridae family, genus flavivirus.[3] However, recently $5^{\text {th }}$ serotype has also been reported in Malaysia during screening of viral samples taken from a 37year-old farmer.[4] Dengue virus is transmitted by mosquito Aedes aegypti and to lesser extent Aedes albopictus. The spread of dengue resembles the Aedes mosquito's geographical distribution, thereby emphasising the role of

'Financial or Other Competing Interest': None.

Submission 28-03-2018, Peer Review 04-05-2018,

Acceptance 11-05-2018, Published 21-05-2018.

Corresponding Author:

Dr. Aroma Oberoi,

Professor and HOD, Department of Microbiology,

Christian Medical College and Hospital,

Ludhiana-141008, Punjab.

E-mail: draromaoberoi@yahoo.com

DOI: $10.14260 /$ jemds $/ 2018 / 575$

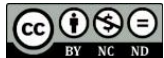

mosquito density in predicting dengue epidemics.[5] DENV is an enveloped single stranded positive sense RNA virus. The RNA genome consists of approximately 10,700 nucleotides and encodes 3,411 amino acids long precursor polyprotein containing three structural proteins (Capsid, precursor membrane and envelope) and seven non-structural (NS) proteins (NS1, NS2A, NS2B, NS3, NS4A, NS4B and NS5).[6,7]

Dengue fever was first reported in India from Vellore district (Tamilnadu) in 1956. The first Dengue haemorrhagic fever (DHF) outbreak occurred in Calcutta (West Bengal) in 1963.[8] Dengue cases reported in India during 2010 were 28,292 , while death of 110 patients were reported. The dengue cases swelled to $1,57,220$ in 2017, while the number of death reported in the same year were 250.[9] The vast majorities of patients who have Dengue fever either remain asymptomatic or have mild symptoms. Few patients have severe form of illness (5\%) and in a small proportion the disease is fatal.[10,11]

Confirmation of dengue infection is done by culture of the virus, Polymerase Chain Reaction (PCR) or serological assays. Culture of the virus is laborious and time-consuming process. Also, the sample must be kept cold and a bio-safety level 3 laboratory is required for the procedure. The prerequisites limit the use of this diagnostic modality in majority of places.[5] Detection of viral RNA from serum, plasma or cells with PCR is based on DENV-specific oligonucleotide primers 
and is fast and robust, although sensitive only in very early stages of disease.[12]

Detection of anti-DENV antibodies (IgM and/ or IgG) is the most commonly used method in all laboratories. The assay technique is simple and various commercial kits are available. The IgM antibody response lasts for a couple of week after infection and the IgG antibodies for several years.[5]

This study was done to find the seropositivity of Dengue (IgM Dengue by ELISA) in blood samples taken from dengue suspected cases.

\section{MATERIALS AND METHODS}

This is a hospital-based descriptive study, conducted at the serology laboratory in the Department of Microbiology at a tertiary care centre from January 2017 to December 2017. Blood samples were received in the department from indoor and outpatients who were suspected to have dengue fever. A total of 1347 samples were collected in 12 months and these samples were processed for IgM Anti-Dengue antibodies (ELISA). The Panbio Dengue IgM Capture ELISA was used for the qualitative detection of IgM antibodies to dengue serotype in serum. Kit instructions, as provided by manufacturer, were strictly followed.

Test principle and result interpretation-Serum antibodies of the IgM class, if present combine with anti-human IgM antibodies attached to the polystyrene surface of the microwell test strips. A concentrated pool of dengue $1-4$ antigens is diluted to the correct working volume with antigen diluents. An equal volume of the HRP conjugated monoclonal antibody (MAb) is added to diluted antigen, which allows the formation of antigen- MAb complexes. Residual serum is removed from the assay plate by washing and complexed antigen- MAb is added to the assay plate. After incubation, the microwells are washed and a colourless substrate system, tetramethylbenzidine (TMB)/ hydrogen peroxide is added. Intensity of colour/ optical density is measured at $450 \mathrm{~nm}$ using the ELISA reader.

\section{RESULTS}

A total of 1347 blood samples of suspected dengue fever were collected and processed between Jan 2017 and Dec 2017. Month wise sample distribution and positivity is depicted in Table 1 . The male-to-female ratio of suspected cases was 1.23: 1 (Fig. 1).

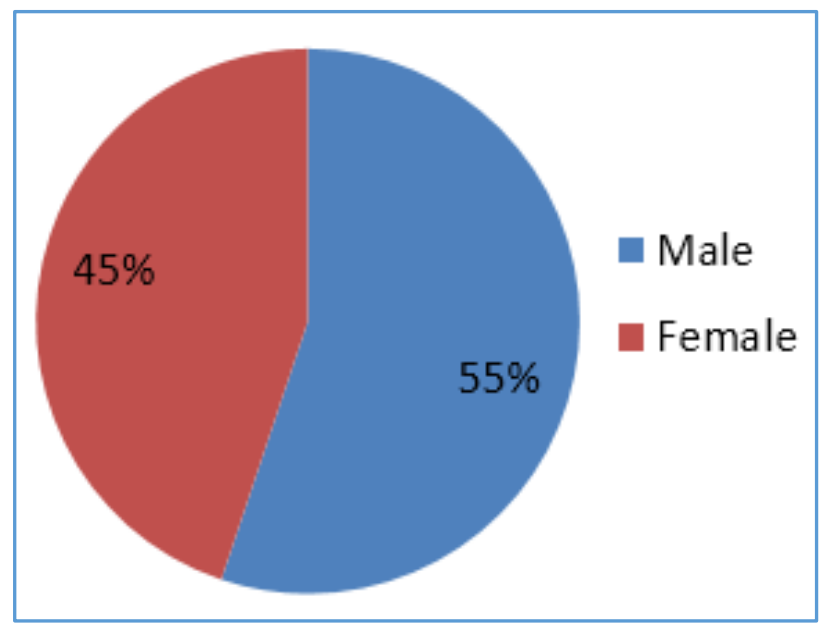

Figure 1. Number of Males and Females in Percentage among suspected Dengue Cases

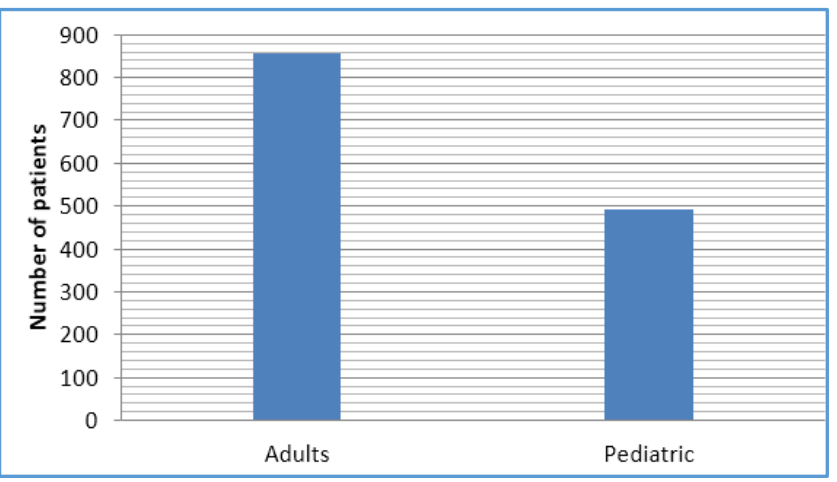

Figure 2. Number of Adults and Paediatric

Patients among suspected Dengue Cases

Of all the samples adults constituted 855 samples, while the remaining 492 were of paediatric population (Fig. 2). IgM Dengue ELISA was found positive in 457 samples (Positivity rate- $33.92 \%$ ) as depicted in Fig. 3. Dengue positive samples were constituted by 316 adults and 141 paediatric patients giving a positivity rate of $36.95 \%$ and $28.65 \%$ respectively. The seropositivity among male and female was $35.12 \%$ and $32.45 \%$ respectively.

\begin{tabular}{|c|c|}
\hline Month (Year) & $\begin{array}{l}\text { No. of Samples Positive for } \\
\text { IgM Dengue (ELISA) }\end{array}$ \\
\hline January (2017) & 01 \\
\hline February (2017) & 01 \\
\hline March (2017) & 0 \\
\hline April (2017) & 0 \\
\hline May (2017) & 0 \\
\hline June (2017) & 02 \\
\hline July (2017) & 01 \\
\hline August (2017) & 11 \\
\hline September (2017) & 60 \\
\hline October (2017) & 208 \\
\hline November (2017) & 161 \\
\hline December (2017) & 12 \\
\hline Total & 457 \\
\hline \multicolumn{2}{|c|}{$\begin{array}{c}\text { Table 1. Month Wise distribution of Dengue Positive } \\
\text { Samples (IgM Dengue by ELISA) }\end{array}$} \\
\hline
\end{tabular}

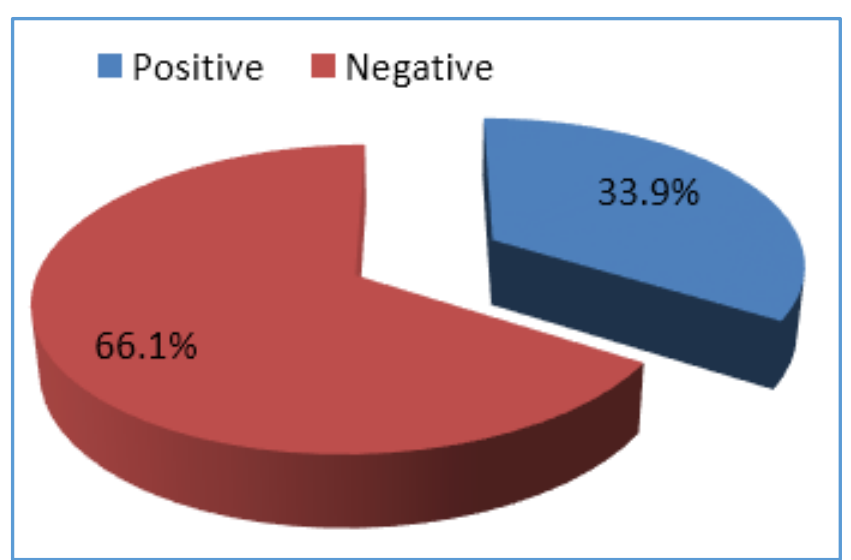

Figure 3. Percentage of Cases detected Positive and Negative among suspected Dengue Cases 


\section{DISCUSSION}

The seroprevalence of Dengue reported in our study is $33.92 \%$. This closely matches the study by PM Ukey et al from Central India, wherein the seroprevalence of dengue was reported as $31.3 \%$.[13] S Padhi et al from Odisha reported the seropositivity of $21.05 \%$ among the patients suspected to have dengue infection.[14] These results are in contrast to the study done by M Kumar et al, who reported to seroprevalence of dengue in Rajasthan as 3.55\%.[15] The main reason behind this low positivity could be firstly as they used Rapid test (in vitro immunochromatographic, one step assay designed to detect IgM and IgG antibodies to dengue) for diagnosis and secondly a large number of non-dengue illness patients also must have been subjected to the testing. ELISA has a greater overall sensitivity than Rapid Diagnostic Tests. In a study, it was observed that overall sensitivity of rapid test in comparison with ELISA was 79.71\%.[16]

Male patients dominated in our study (M: F ratio=1.23:1), which is similar to study done in various parts of India.[13,14,15] This pattern indicates that men are more susceptible as majority of men work outside during the day and Aedes mosquitoes bite during the day only. However, one study reported female preponderance among dengue positive cases.[17]

The maximum number of dengue positivity was detected during the post monsoon period (Maximum in October followed by November and September respectively). Similar findings were given by $\mathrm{H}$ Lall et al, who observed that the highest numbers of cases were recorded between August and November.[17] The number of affected cases declined with the onset of winter (December onwards).[17] PM Ukey et al reported that the highest number of suspected dengue patients admitted was in the month of November (2005) followed by in October.[13] From January 2006 to August 2006, out of 16 suspected sera no sample was found positive for dengue antibodies.[1] Also S Padhi has a similar observation, wherein the majority (47.86\%) of cases was detected in the month of September.[14] The reason put up by majority of authors for this active viral transmission during monsoon and post-monsoon season is that Aedes aegypti vector mosquito breeds during the rainy season and continues to reproduce and add to its number usually till the winter season.

The limitation of study is that enzyme-linked immunosorbent assay (ELISA) for IgM Anti-Dengue antibodies were not compared with gold standard eg. virus isolation. Secondly, even NS1 antigen detection by ELISA should have been done as few cases who presented very early in disease course would have been missed as IgM antiDengue antibodies are absent in first few days.

\section{CONCLUSION}

Dengue infection is rapidly growing in India with each passing year. Prompt diagnosis is the key to better management and decreased morbidity/mortality. In our study, a commercial kit (Panbio IgM Dengue ELISA) was used for diagnosis of dengue fever. Various steps at multiple levels (Curb mosquito breeding, early diagnosis and treatment) are needed to stop the growing number.

\section{REFERENCES}

[1] Guzman MG, Halstead SB, Artsob H, et al. Dengue: a continuing global threat. Nat Rev Microbiol 2010;8(Suppl 12):S7-S16.

[2] Guzmán MG, Kourí G. Dengue: an update. Lancet Infect Dis 2002;2(1):33-42.

[3] Westaway EG, Brinton MA, Gaidamovich SY, et al. Flaviviridae. Intervirology 1985;24(4):183-92.

[4] Mustafa MS, Rasotgi V, Jain S, et al. Discovery of fifth serotype of dengue virus (DENV-5): a new public health dilemma in dengue control. Med J Armed Forces India 2015;71(1):67-70.

[5] WHO. Dengue haemorrhagic fever. Diagnosis, treatment, prevention and control. 2nd edn. Geneva: WHO 1997: p. 12-23.

[6] Pang X, Zhang M, Dayton AI. Development of Dengue virus type 2 replicons capable of prolonged expression in host cells. BMC Microbiol 2001;1:18.

[7] Alvarez DE, Lodeiro MF, Filomatori CV, et al. Structural and functional analysis of dengue virus RNA. Novartis Found Symp 2006;277:120-32.

[8] Baruah K, Dhariwal AC. Epidemiology of dengue, its prevention and control in India. J Indian Med Assoc 2011;109(2):82-6.

[9] Dengue/DHF situation in India - NVBDCP. nvbdcp.gov.in

[10] Whitehorn J, Farrar J. Dengue. Br Med Bull 2010;95(1):161-73.

[11] Reiter P. Yellow fever and Dengue: a threat to Europe? Euro Surveill 2010;15(10):19509.

[12] Rigau-Perez JG, Clark GG, Gubler DJ, et al. Dengue and Dengue haemorrhagic fever. Lancet 1998;352(9132):971-7.

[13] Ukey PM, Bondade SA, Paunipagar PV, et al. Study of seroprevalence of Dengue fever in central India. Indian J Community Med 2010;35(4):517-9.

[14] Padhi S, Dash M, Panda P, et al. A three year retrospective study on the increasing trend in seroprevalence of Dengue infection from southern Odisha, India. Indian J Med Res 2014;140(5):660-4.

[15] Kumar M, Sharma R, Parihar G, et al. Seroprevalence of Dengue in central Rajasthan: a study at a tertiary care hospital. Int J Curr Microbiol App Sci 2015;4(9):93340.

[16] Gill MK, Kaur A, Kukreja S, et al. Comparative evaluation of a rapid test with ELISA for the detection of Dengue infection. Indian J Microbiol Res 2016;3(4):405-7.

[17] Lall H, Gupta P, Debbarma M, et al. Sero-Prevalence of Dengue in tertiary care hospital in Delhi. Int J Curr Microbiol App Sci 2016;5(6):439-45. 